\title{
camensaum \\ Intensified therapy for Ewing sarcoma
}

Administering chemotherapy every 2 weeks, rather than every 3 weeks, can improve treatment efficacy without an increase in toxicity in patients with Ewing sarcoma, according to a new study published in Journal of Clinical Oncology.

Ewing sarcoma is a rare aggressive malignancy of the bone, which mainly affects children or adolescents. Current treatment of localized Ewing sarcoma involves alternating cycles of vincristinedoxorubicin-cyclophosphamide (VDC) and ifosfamide-etoposide (IE) plus surgery and/or radiotherapy of the primary tumour; about $70 \%$ of patients with localized disease can be cured with this therapy. The Children's Oncology Group has investigated whether intensifying chemotherapy by reducing the intervals between each cycle would improve outcome.

In this prospective, controlled study, 568 eligible patients (aged $<50$ years) with newly diagnosed localized Ewing sarcoma were randomly assigned to receive chemotherapy (alternating cycles of VDC and IE combined with filgrastim, a granulocyte colony-stimulating factor analogue) every 21 days or 14 days. The mean cycle durations were $22.45 \pm 4.87$ days and $17.29 \pm 5.40$ days in each arm, respectively. Both groups also underwent surgery, radiotherapy, or both, for treatment of the primary tumour at week 13 of chemotherapy. Patients were followed up for a median of 61 months (range 7-94 months) and the primary end point was event-free survival (EFS), defined as time from enrolment until the time of incidence of an adverse event (disease progression, metastasis or death).

The EFS at 5 years was $73 \%$ for patients receiving intensified chemotherapy (every 14 days) and $65 \%$ for those receiving therapy every 21 days, accounting for a $22 \%$ reduction in the risk of recurrence. Overall survival was $83 \%$ and $77 \%$ for each arm, respectively. Importantly, intensified therapy was generally well tolerated, with no increased toxicity.

The investigators are now interested in testing the intensification strategy in other

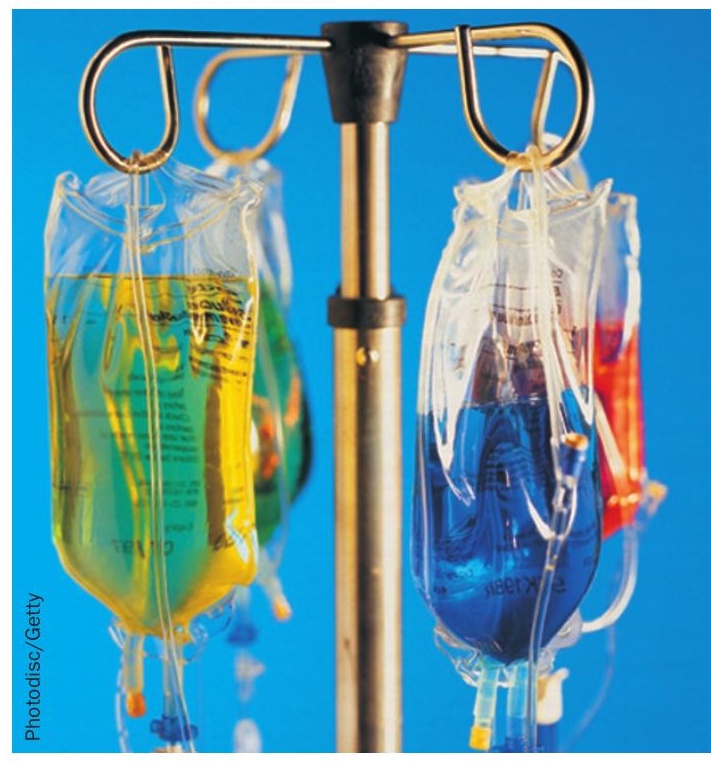

types of sarcoma. More work is required to understand the mechanisms behind improved efficacy with intensified therapy.

Iley Ozerlat

Original article Womer, R. B. et al. Randomized controlled trial of interval-compressed chemotherapy for the treatment of localized Ewing sarcoma: a report from the Children's Oncology Group. J. Clin. Oncol. doi: 10.1200/ JC0.2011.41.5703 